\title{
Diabetic peripheral neuropathy and sudomotor dysfunction in Saudi patients with newly diagnosed type 2 diabetes mellitus
}

\begin{abstract}
Using the Toronto Diabetic Neuropathy Expert Group-2010 classification of diabetic peripheral neuropathy (DPN), $89 \%$ of patients with newly diagnosed type 2 diabetes had either confirmed or subclinical-DPN, hence at increased risk of foot complications. Sudomotor-dysfunction (SMD) assessed through electrochemical skin-conductance measurement was present in $51.1 \%$. SMD has $73 \%$ sensitivity and $81 \% \mathrm{NPV}$ to detect confirmed-DPN.
\end{abstract}

Keywords:diabetic peripheral neuropathy, sudomotor dysfunction, newly diagnosed type 2 diabetes mellitus, electrochemical skin conductance, nerve conduction study
Volume 4 Issue 2 - 2017

\author{
Eman Sheshah, Fahad Algreeshah, Khaled Al- \\ Madani, Dalal Al-Qaisi,Amal Madanat \\ Diabetes Care Center, King Salman Hospital, Saudi Arabia
}

Correspondence: Amal Madanat, PO Box 17003,Amman I I I 95, Saudi Arabia, Tel +96 279554 2322,

Email madanatamal.y@gmail.com

Received: February 22, 2017 | Published: April 07, 2017
Abbreviations: DPN, diabetic peripheral neuropathy; SMD, sudomotor dysfunction; DM, diabetes mellitus; N-T2DM, newly diagnosed type 2 diabetes mellitus; FU, foot ulceration; TDNEG-2010, toronto diabetic neuropathy expert group-2010; NS, neuropathic symptoms; NDS, neuropathy disability score; NSS, neuropathy symptom score; NCS, nerve conduction study; LFDPN, large fiber-DPN; AAN, american academy of neurology; ESC, electrochemical skin conductance; OR, odds ratio; ROC, receiver operator characteristics; SPSS, statistical package for social sciences

\section{Introduction}

The prevalence of Type 2 diabetes mellitus (T2DM) is increasing worldwide including in Saudi Arabia. ${ }^{1,2}$ About $46 \%$ of people with T2DM may remain undiagnosed for many years, with the result that patients can already display diabetes microvascular complications at time of diagnosis. ${ }^{2}$ Diabetic peripheral neuropathy (DPN) is the earliest and most common long term complications of DM; it leads directly to increased risk foot ulceration (FU), limb loss and economic burden. ${ }^{3}$ Rates of DPN in newly diagnosed T2DM (N-T2DM) vary considerably, ranging from $2.4 \%$ to $29.2 \%$ and up to $68.1 \% .^{4-9}$ Contributing factors for such variation might include different ethnicities, diversity in the types of tests used to detect DPN and disparities in health care delivery systems. Sudomotor-dysfunction (SMD) is associated with increased risk of FU in T2DM patients and may occur in patients with impaired glucose tolerance or in N-T2DM. ${ }^{10,11}$ The aims of the study were to explore the occurrence of neuropathic symptoms (NS), DPN and SMD in Saudi patients with N-T2DM, and to test the performance of SMD to screen for DPN.

\section{Materials and methods}

\section{Study subjects}

This is a cross-sectional study conducted on 125 Saudi patients with N-T2DM (i.e. $<6$ months) in a diabetes center between January and May 2013. The study was approved by the hospital's local Ethics Committee and written informed consent was obtained. Patients with secondary causes of neuropathy, peripheral vascular disease, active FU, critical illness or on medications that could potentially affect symptoms or sudomotor function testing results were excluded.

\section{Clinical assessment}

Feet assessment was performed per Boulton. ${ }^{12}$ Neuropathic symptoms were assessed using neuropathy-symptom-score (NSS) and considered positive if $\mathrm{NSS} \geq 5 .{ }^{13}$ The presence of DPN was determined using the neuropathy-disability-score (NDS) of Abbott. ${ }^{13}$ Scores were derived from testing motor and sensory function using: tendon hammer, $128-\mathrm{Hz}$ tuning fork (128-Hz TF), and neurotip, warm and cool rod. ${ }^{13}$ DPN was present if NDS $\geq 3$.

\section{Neurophysiologic assessment}

Nerve conduction study (NCS) was performed in 87 participants for the right and left Sural, Peronial and Tibial nerves using Nicolet Viking Quest VIAsys-USA. Large fiber-DPN (LF-DPN) was present if the participant had at least one symptom or sign of DPN and one or more abnormal nerve conduction tests in both Sural (sensory) and Peroneal or Tibial (motor) nerves in accordance with the case definition of peripheral neuropathy described by the American Academy of Neurology (AAN). ${ }^{14}$ Further sub-division to subclinical or confirmed LF-DPN was performed as described by the Toronto Diabetic Neuropathy Expert Group (TDNEG-2010). ${ }^{15}$

\section{Sudomotor-function testing}

Hands and feet electrochemical-skin-conductance (ESC) was assessed in 92 participants using Sudoscan. As hands-ESC correlated significantly with feet-ESC, we used only data for feet-ESC. Sudomotor dysfunction was absent if feet-ESC $>70 \mu \mathrm{S}$, moderate if feet-ESC $<70->50 \mu \mathrm{S}$, severe if feet-ESC $<50 \mu \mathrm{S} .{ }^{16}$

\section{Statistical analysis}

Data are presented as mean SD or percentages. Variances between variables were calculated using an independent T-test. Logistic regression analysis was used to estimate the odds ratio (OR) of 
having DPN according to SMD. ROC curve analysis, sensitivity and specificity were used to access SMD performances to detect DPN. For all tests a P-value of 0.05 or less was used for statistical significance. Statistical Package for Social Sciences (SPSS) v. 20 for windows was used for statistical analysis.

\section{Results}

Clinical characteristics of the participants are presented in Table 1. Neuropathic symptoms defined by NSS $\geq 5$ were present in (44) $35.2 \%$ and DPN defined by NDS $\leq 3$ in (11) $8.8 \%$. Among the 87 participants in whom neurophysiologic assessment was performed, subclinical and confirmed LF-DPN classified as described by the TDNEG-2010 15 were present in (44) $50.6 \%$ and (34) $39 \%$ respectively and only (9) $10.4 \%$ did not have LF-DPN. In those 87 participants, neuropathic symptoms defined by $\mathrm{NSS} \geq 5$ and DPN defined by NDS $\leq 3$ were present in (29) 33\% and in (8) 9.1\% respectively. Moderate and severe SMD according to feet-ESC were present in 30(34.8\%), 15(16.3\%) respectively. Percentages of patients with SMD in participants with confirmed LF-DPN as compared to those with subclinical or without DPN are displayed in Figure 1. Using the TDNEG-2010 classification for confirmed LF-DPN as reference for ROC curve analysis, SMD (feet-ESC $<70 \mu \mathrm{S}$ ) had an AUC of 0.71 for detection of confirmed LFDPN with a sensitivity of $73 \%$ and a negative predictive value (NPV) of $81 \%$. Using feet $\mathrm{ESC} \geq 70 \mu \mathrm{S}$ as reference, patients with feet-ESC between $50-70 \mu \mathrm{S}$ or $<50 \mu \mathrm{S}$ had an OR-adjusted for gender, age, BMI, treatment of diabetes, dyslipidemia and smoking status- of 4.87(1.01$23.5)$ and $12.4(1.90-81.0)$ respectively for having confirmed LF-DPN. The OR for each group was 4.97(1.58-15.6) and 6.07(1.52-24.3) respectively for having NS according to NSS $\geq 5$.

Table I Clinical Characteristic of the Participants

\begin{tabular}{llllllllllllll}
\hline No & Age & \multicolumn{3}{l}{ Gender } & \multicolumn{2}{c}{ Treatment of DM } & & & BMI & Smoking & HbAlc & HTN & DLP \\
\hline $100 \%$ & Years & M\% & F\% & None\% & OHA $\%$ & Insulin\% & Combined\% & $\mathrm{Kg} / \mathrm{m}^{2}$ & $\%$ & $\%$ & $\%$ & $\%$ \\
125 & $45.4 \pm 10.3$ & 58.5 & 41.5 & 4 & 91.2 & 0.8 & 4 & $31.6 \pm 7.0$ & 15.2 & $9.5 \pm 2.4$ & 31.2 & 48.8 \\
\hline
\end{tabular}

M, male; F, female; OHA, oral hypoglycemic agents; BMI, body mass index; His, history of foot ulcer; HbAlc, glycated hemoglobin;

HTN, hypertension; DLP, dyslipidemia

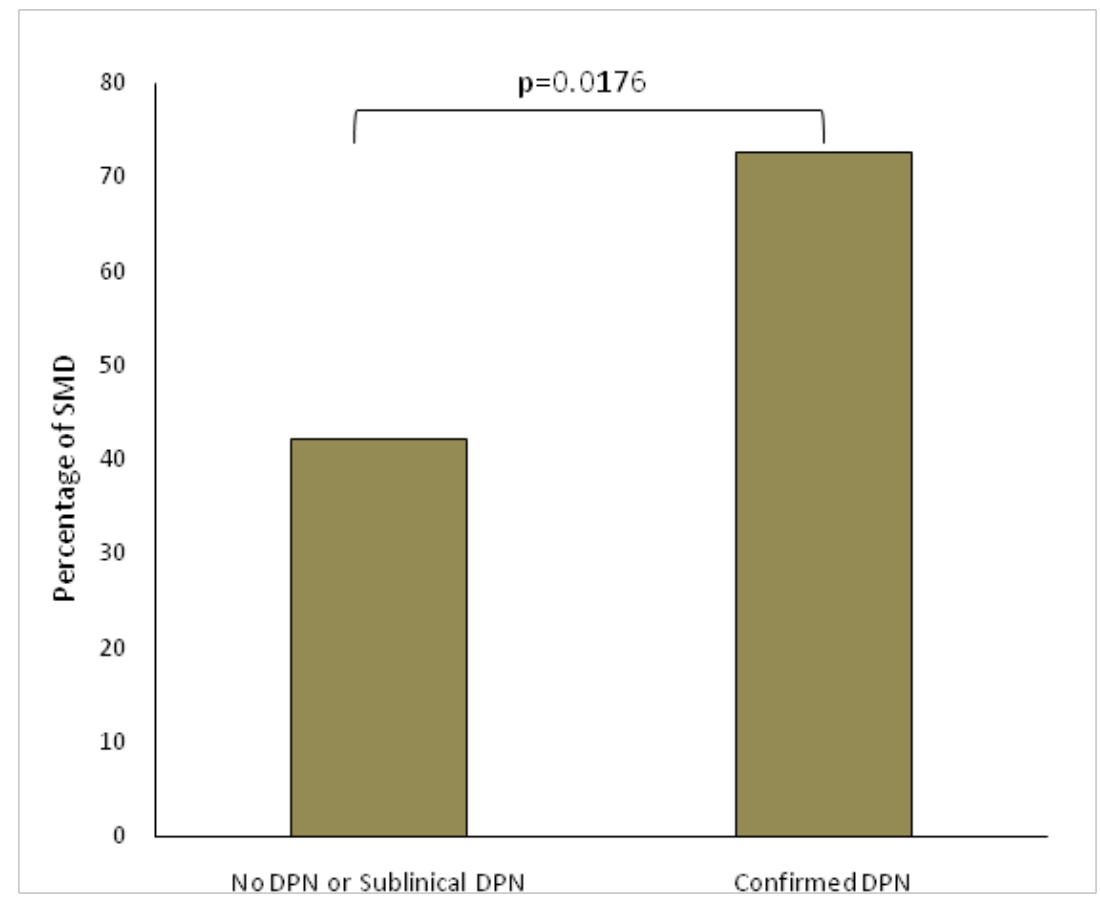

Figure I The presence of sudomotor dysfunction (Feet ESC $<70 \mu S$ ) in percentages among participants with confirmed LF-DPN compared to participants without LF-DPN or with subclinical LF-DPN (n:68).

\section{Discussion}

Our study demonstrated that $89 \%$ of the participants with N-T2DM had subclinical or confirmed LF-DPN diagnosed objectively by NCS, thus they were at increase risk of foot complications early in the course of the disease. Earlier Al-Sulaiman et al. ${ }^{17}$ in a study on 29 Saudi patients with N-T2DM (within 4weeks from the diagnosis) demonstrated that almost all of the participants had NCS abnormalities in both sensory and motor peripheral nerves. When traditional bedside tests that are included in the NDS were used to detect DPN, the number of affected patients by DPN in our study decreased dramatically to $9.1 \%$, leaving many at-risk patients unidentified and unattended. Previously Dyke et al. ${ }^{18}$ have found that traditional bedside tests to detect DPN are subjective and identify patients with DPN when it is well established.

We also demonstrated that about half of the participants have SMD and that this occurred in parallel with confirmed LF-DPN in 
about $70 \%$ of the participants with confirmed LF-DPN as shown in the Figure 1. Additionally, SMD assessed by feet-ESC exhibited high sensitivity and NPV in screening of confirmed LF-DPN. These results suggest a potential role for feet-ESC measurement to screen patients with N-T2DM not only for SMD, but for generalized DPN as well. Measurement of feet-ESC is simple, objective and may enable identification of more patients at risk of developing foot complications. The study has limitations: it is cross-sectional, performed in one center and limited in its number of participants.

\section{Conclusion}

The study demonstrates high occurrence of NS, DPN and SMD in NT2DM Saudi patients. There is a need for utilizing simple objective tools to detect DPN at diagnosis of T2DM. Further studies on a larger population of patients to confirm the findings are warranted.

\section{Acknowledgments}

The authors wish to express gratitude to Nurses in the Neuro-Lab: Eman Al-Zahrani and Reem El-Esmary. Part of the study results were presented as a poster during the World Diabetes Congress, IDF, 30 November - 4 December 2015, Vancouver BC, Canada.

\section{Funding}

This research did not receive any specific grant from funding agencies in the public, commercial, or not-for-profit sectors.

\section{Authors' contribution}

ES participated in the study design, researched data and draft review. FG participated in supervising neurophysiologic testing and draft review. KM participated in data acquisition and draft review. DQ participated in data acquisition and draft review. AM participated contributed to the concept and design of the study, researched data, performed the statistical analysis and drafted the manuscript.

\section{Conflict of interest}

Author declares that there is no conflict of interest.

\section{References}

1. International Diabetes Federation. IDF Diabetes Atlas, 7th ed. Belgium: International Diabetes Federation. 2015.

2. AL Dawish MA, Robert AA, Braham R, et al. Diabetes mellitus in Saudi Arabia: a review of the recent literature. Curr Diabetes Rev. 2016;12(4):359-368.

3. Vinik AI , Mitchell BD, Leichter SB, et al. Epidemiology of the complications of diabetes. In: Leslie RDG, Robbins DC, editors. Diabetes: Clinical Science in Practice. Cambridge: Cambridge University Press; 1994. p. 221-287.

4. Kostev K, Jockwig A, Hallwachs A, et al. Prevalence and risk factors of neuropathy in newly diagnosed type 2 diabetes in primary care practices: a retrospective database analysis in Germany and UK. Prim Care Diabetes. 2014;8(3):250-255.
5. Gill HK, Yadav SB, Ramesh V, et al. Prospective study of prevalence and association of peripheral neuropathy in Indian patients with newly diagnosed type 2 diabetes mellitus. JPostgrad Med.2014;60(3):270-275.

6. Sosale A, Prasanna Kumar KM, Sadikot SM, et al. chronic complications in newly diagnosed patients with Type 2 diabetes mellitus in India. Indian J Endocrinol Metab. 2014;18(3):355-360.

7. Ali A, Iqbal F, Taj A, et al. Prevalence of microvascular complications in newly diagnosed patients with type 2 diabetes. Pak J Med Sci. 2013;29(4):899-902.

8. Harzallah F, Ncibi N, Alberti $\mathrm{H}$, et al. Clinical and metabolic characteristics of newly diagnosed diabetes patients: experience of a university hospital in Tunis. Diabetes Metab. 2006;32(6):632-635.

9. Spijkerman AM, Dekker JM, Nijples G, et al. Microvascular Complications at Time of Diagnosis of Type 2 Diabetes Are Similar Among Diabetic Patients Detected by Targeted Screening and Patients Newly Diagnosed in General Practice. The Hoorn Screening Study. Diabetes Care. 2003;26(9):2604-2608.

10. Tentolouris N, Marinou K, Kokotis P, et al. Sudomotor dysfunction is associated with foot ulceration in diabetes. Diabet Med. 2009;26(3):302-305.

11. McDaid EA, Monaghan B, Parker AI, et al. Peripheral autonomic impairment in patients newly diagnosed with type II diabetes. Diabetes Care. 1994;17(12):1422-1427.

12. Boulton AJ, Armstrong DG, Albert SF, et al. Comprehensive foot examination and risk assessment: a report of the Task Force of the Foot care Interest Group of the American Diabetes Association, with endorsement by the American Association of Clinical Endocrinologists. Dibetes Care. 2008;31(8):1679-1685.

13. Abbott CA, Carrington AL, Ashe H, et al. The North-West Diabetes Foot Care Study: incidence of, and risk factors for, new diabetic foot ulceration in a community based patient cohort. Diabet Med. 2002;19(5):377-384.

14. England JD, Gronseth GS, Franklin G, et al. Distal Symmetrical Polyneuropathy: Definition for Clinical Research: report of the American Academy of Neurology, the American Association of Electrodiagnostic Medicine and the American Academy of Physical Medicine and Rehabilitation. Neurology. 2005;64(2):199-207.

15. Tesfaye S, Boulton AJ, Dyck PJ, et al. Diabetic Neuropathies: Update on definitions, diagnostic criteria, estimation of severity, and treatments. Diabetes Care. 2010;33(10):2285-2293.

16. Vinik AI, Smith AG, Singleton JR, et al. Normative Values for Electrochemical Skin Conductances and Impact of Ethnicity on Quantitative Assessment of Sudomotor Function. Diabetes Technol Ther. 2016;18(6):391-398.

17. Al-Sulaiman AA, Ismail HM, Al-Sultan AI. Electrophysiological findings in newly diagnosed Non-Insulin Dependent Diabetes: A prospective study. Ann Saudi Med. 1997;17(4):399-401.

18. Dyck PJ, Overland CJ, Low PA, et al. Signs and symptoms versus nerve conduction studies to diagnose diabetic sensorimotor polyneuropathy: Cl vs. NPhys trial. Muscle Nerve. 2010;42(2):157-164. 